\title{
Conformal operators for partially massless states
}

\section{Louise Dolan}

Department of Physics, University of North Carolina

Chapel Hill, NC 27599-3255, USA

E-mail: doìä@physics.unc.edü

\section{Chiara R. Nappi}

Department of Physics, Jadwin Hall, Princeton University

Princeton, NJ 08540, USA

E-mail: nappioịas.edü'

\section{Edward Witten}

School of Natural Sciences, Institute for Advanced Study

Olden Lane, Princeton, NJ 08540, USA

E-mail: wîtten@ias.edü

AbstRACT: The AdS/CFT correspondence is explored for "partially massless" fields in AdS space (which have fewer helicity states than a massive field but more than a conventional massless field). Such fields correspond in the boundary conformal field theory to fields obeying a certain conformally-invariant differential equation that has been described by Eastwood et al. The first descendant of such a field is a conformal field of negative norm. Hence, partially massless fields may make more physical sense in de Sitter as opposed to Anti de Sitter space.

KEYwords: iĀ 


\section{Contents}

ii. Introduction

2. Review of ingredients

'2.1' Partially massless field equations

2.-12 Metric near the boundary of AdS space

2.3. Partially conserved conformal operators

3. Applying the AdS/CFT correspondence to a partially massless field

:

4. Conformal algebra and unitary conformal field theory 6

75. A cosmological solution

\section{Introduction}

In four-dimensional Minkowski space, a massless field of spin $s$ has helicities $\pm s$, while a massive field has all possible helicities $-s,-s+1, \ldots, s$. When the cosmological constant is non zero, however, the range of possibilities is greater [i, $\left.\overline{1}_{1}\right]-\left[\begin{array}{l}1 \\ -1\end{array}\right]$. In addition to the familiar massless and massive fields, one can have "partially massless" fields whose helicity ranges over the set $-s,-s+1, \ldots, s$ with $-n,-n+1, \ldots, n$ removed, for any $n \leq s-2$. The mass squared of such a field is

$$
m^{2}=\frac{\Lambda}{3}(s(s-1)-n(n+1)) .
$$

Here we define $\Lambda$ by writing the Einstein equation as $R_{\mu \nu}=\Lambda g_{\mu \nu}$.

A partially massless field is described by a symmetric tensor field $\phi_{\mu_{1}, \ldots, \mu_{n}}$ with a gauge invariance

$$
\delta \phi_{\mu_{1} \mu_{2} \cdots \mu_{s}}=D_{\mu_{1}} \cdots D_{\mu_{s-n}} \xi_{\mu_{s-n+1} \cdots \mu_{s}}+\cdots,
$$

where the $+\cdots$ refers to terms obtained by symmetrizing the indices and adding additional contributions with fewer than $s-n-1$ derivatives. We will describe later in more detail the first non-trivial case, with $s=2$ and $n=0$.

Formally, partially massless fields can be defined for either positive or negative cosmological constant. We note, however, from ('i $\left.\mathbf{1}_{1} \overline{1}_{1}^{\prime}\right)$ that for $\Lambda<0$, a partially massless field has negative mass squared, smaller than that of a massless field which has more gauge invariance - a result that seems unintuitive. By contrast, for $\Lambda>0$, the partially massless field has a positive mass squared. 
In this paper, we will study partially massless fields for $\Lambda<0$ using the AdS/CFT correspondence. Just as a massless field of spin $s$ corresponds in the boundary theory to a rank $s$ conserved symmetric tensor, a partially massless field must correspond in the boundary theory to a partially conserved tensor. Such a tensor is a field $L^{\mu_{1} \cdots \mu_{s}}$, symmetric in its indices, and obeying a conformally invariant equation whose general form we can guess given (1, $\left.1 \overline{2}_{-1}^{\prime}\right)$. Since a coupling $\int L^{\mu_{1} \cdots \mu_{s}} \phi_{\mu_{1} \cdots \mu_{s}}$ should be gauge-invariant, the equation must have the form

$$
D_{\mu_{1}} D_{\mu_{2}} \cdots D_{\mu_{s-n}} L^{\mu_{1} \cdots \mu_{s}}+\cdots=0
$$

where the $+\cdots$ refers to symmetrization of indices and terms of lower order that are proportional to the curvature tensor of the boundary. This equation must be conformally invariant, since the boundary of AdS space has only a conformal structure, not a metric. In fact, the appropriate conformally invariant differential equations have been described in a series of papers [1] of (1..3in $)$, starting with a partially massless field in the bulk, for the first non-trivial case of $s=2, n=0$; the same idea should work in general.

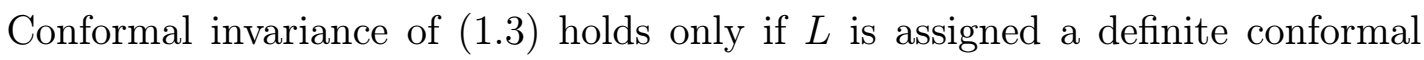
dimension. This corresponds in the AdS/CFT correspondence to the fact that the partially massless field has a definite $m^{2}$ given in (1: $\left.\overline{1}_{1}^{1}\right)$; from that point of view, the conformal dimension of $L$ is determined, in a standard fashion, from the behavior of $\phi$ near the boundary of AdS space.

A third way to determine the conformal dimension of $L$ is as follows. Via the operator-state correspondence of conformal field theory, the field $L^{\mu_{1}, \ldots, \mu_{s}}$ corresponds to a state $\left|\Psi^{\mu_{1}, \ldots, \mu_{s}}\right\rangle$, which (given that $L$ transforms covariantly under Weyl transformations) must be a highest weight vector for the conformal group. Equation (1. for $L$ means that a certain level $s-n$ descendant of $|\Psi\rangle$ is a null vector. This occurs only for a particular conformal dimension for $L$, which can be conveniently computed using radial quantization, and agrees with the results obtained by other methods.

In sum, then, this paper is devoted to the correspondence between three types of objects:

(A) A partially massless field $\phi$ in AdS space.

(B) A field $L$ on the boundary of AdS space that obeys the conformally invariant eq. ('in $\overline{1} \cdot \overline{3} i)$.

(C) A highest weight vector $|\Psi\rangle$ of the conformal group in a representation that has a certain null vector at level $s-n$.

Our analysis of $(C)$ also shows that a certain descendant of $|\Psi\rangle$ at a lower level has negative norm. Despite the nice consistency between $(A),(B)$, and $(C)$, this seems discouraging for most physical applications of partially massless fields 
in the AdS case. This may correspond to the strange sign of (1.1.1. Alternatively, we could consider partially massless fields in de Sitter space, with $\Lambda>0$, where (1, $\left.\overline{1}_{i}^{\prime}\right)$ has a more intuitive sign. In this case, many considerations of the AdS/CFT correspondence can be imitated [1] i, 'i 18 in though the physical meaning is less clear. Our computations relating $(A),(B)$, and $(C)$ are purely formal, so they make sense for $\Lambda>0$. For de Sitter space, the boundary conformal field theory (if that is the right notion) is anyway not unitary [1] $\left.\overline{1}_{-1}\right]$, so the negative norm descendant is not a problem.

In this paper, we consider only the case of AdS space with bulk dimension $D=4$ and boundary dimension $d=D-1=3$, though the arguments presumably generalize to any dimension. In particular, the conformally invariant eqs. (i- (i)

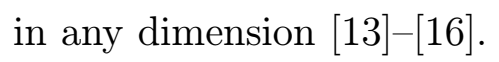

In the last section, we present a cosmological solution of the boundary conformal field theory which relates the time dependence of the boundary operator to the Hubble function.

\section{Review of ingredients}

\subsection{Partially massless field equations}

First, we recall explicitly the field equation of a partially massless field of spin two and $n=0$, in four dimensions.

We denote as $g_{\mu \nu}$ a background metric that obeys the Einstein equations

$$
R_{\mu \nu}=\Lambda g_{\mu \nu}
$$

and we let $C_{\alpha \mu \nu \rho}$ denote the Weyl tensor. The Riemann and Ricci tensors are defined by $R_{\mu \nu \lambda}^{\rho} \equiv \partial_{\nu} \Gamma_{\mu \lambda}^{\rho}-\partial_{\lambda} \Gamma_{\mu \nu}^{\rho}+\Gamma_{\mu \lambda}^{\sigma} \Gamma_{\sigma \nu}^{\rho}-\Gamma_{\mu \nu}^{\sigma} \Gamma_{\sigma \lambda}^{\rho}$, and $R_{\mu \nu} \equiv R_{\mu \lambda \nu}^{\lambda}$, and $C$ is obtained from the Riemann tensor by subtracting trace terms.

The spin two partially massless field is a symmetric tensor $\phi_{\mu \nu}$ whose field equation in $D$ dimensions, in a background Einstein spacetime, reads

$$
\left(\square-\frac{D \Lambda}{(D-1)}\right) \phi_{\mu \nu}-D_{\mu} D_{\nu} \phi_{\rho}^{\rho}+\frac{\Lambda}{(D-1)} g_{\mu \nu} \phi_{\rho}^{\rho}-2 C_{\alpha \mu \nu \rho} \phi^{\alpha \rho}=0 .
$$

A single divergence of the field equation gives the constraint $D^{\mu} \phi_{\mu \nu}-D_{\nu} \phi_{\rho}^{\rho}=0$. The equations of motion have a scalar gauge invariance given by

$$
\phi_{\mu \nu} \longrightarrow \phi_{\mu \nu}+D_{\mu} D_{\nu} \xi+\frac{\Lambda}{(D-1)} g_{\mu \nu} \xi
$$

The precise form of (2.2. gauge invariance, one makes use of (는. 
A non-zero $\phi$ field has a non-zero stress tensor, and so physically, in the presence of such a field, one should no longer impose the vacuum Einstein equations (2. $\left.\overline{1}_{1}^{\prime}\right)$; there should be additional contributions quadratic in $\phi$. In the presence of such terms, the proof of gauge invariance of $\left(2.2_{2}^{2}\right)$ does not work, so one must add additional contributions to (2.2. law for the metric, $\delta g \sim \epsilon \phi^{2}$. It has not been demonstrated in the literature that gauge-invariance of the $\phi$ field can be maintained exactly (beyond linear order in $\phi)$. However, we will proceed assuming that a fully non-linear theory of partially massless fields does exist. Assuming that the higher order terms can be chosen to maintain gauge invariance, their details will not concern us.

\subsection{Metric near the boundary of AdS space}

We take $\Lambda<0$, and consider an Einstein manifold that is asymptotic near its conformal boundary — which we take to be at $u=0$ — to AdS space. With a suitable choice of coordinates, the metric $g_{\mu \nu}$ of such a manifold can be expressed as

$$
d s^{2}=-\frac{(D-1)}{\Lambda} u^{-2}\left(d u^{2}+f_{i j}(u, x) d x^{i} d x^{j}\right)
$$

where the expansion of $f_{i j}(u, x)$ in powers of $u$ is determined from the Einstein equations, and is found $[\underline{1} \overline{1} \overline{9}]-[\overline{2} \overline{2}]$ to be, to the order that we will need,

$$
f_{i j}(u, x)=\tilde{g}_{i j}-u^{2}(d-2)^{-1}\left(\tilde{R}_{i j}-\frac{1}{2(d-1)} \tilde{R} \tilde{g}_{i j}\right)+O\left(u^{3}\right)
$$

when $d=D-1$. The conformal metric on the boundary $(u=0)$ is $\tilde{g}_{i j}$. We do not assume that it is conformally flat or conformally Ricci-flat.

\subsection{Partially conserved conformal operators}

As explained in the introduction, the partially massless field $\phi$ will correspond in the boundary to a symmetric tensor field $L^{i j}$ that will obey a conformally invariant "partial conservation law." The requisite conformally invariant equation, which is a special case of the conformally invariant differential equations studied in [1] 13 i in $d$ dimensions

$$
D_{i} D_{j} L^{i j}+\frac{1}{(d-2)} \tilde{R}_{i j} L^{i j}=0 .
$$

One can verify directly that this equation is invariant under the Weyl transformation law

$$
\begin{aligned}
\tilde{g}_{i j}(x) & \longrightarrow e^{-2 \sigma(x)} \tilde{g}_{i j}(x), \\
L^{i j}(x) & \longrightarrow e^{(d+1) \sigma(x)} L^{i j}(x),
\end{aligned}
$$

since under $\left(\overline{2}_{2} \overline{7}_{1}\right)$ the Ricci tensor transforms as $\tilde{R}_{i j} \rightarrow \tilde{R}_{i j}+(d-2)\left(D_{i} D_{j} \sigma+\right.$ $\left.D_{i} \sigma D_{j} \sigma\right)+\tilde{g}_{i j}\left(D^{k} D_{k} \sigma-(d-2) D_{k} \sigma D^{k} \sigma\right)$, and $D_{i} D_{j} L^{i j} \rightarrow e^{(d+1) \sigma}\left[D_{i} D_{j} L^{i j}-L^{i j}\left(D_{i} D_{j}\right.\right.$ 
$\left.\left.\sigma+D_{i} \sigma D_{j} \sigma\right)\right]$, and $L^{i j}$ is traceless. Note that by successively lowering indices, we get fields $L_{j}^{i}$ and $L_{i j}$ with different Weyl transformation laws, i.e. $L_{j}^{i} \rightarrow e^{(d-1) \sigma} L_{j}^{i}$. The weight in the Weyl transformation law of the field with half its indices up and half down is called the conformal dimension in conformal field theory. So $L$ corresponds to a field of conformal dimension $d-1$, and thus of dimension 2 if $d=3$.

\section{Applying the AdS/CFT correspondence to a partially massless field}

Now we will make the correspondence between $(A)$ and $(B)$ as described in the introduction: we will show that a partially massless spin two field in the bulk of AdS space corresponds to a conformal field on the boundary that obeys the partial conservation law. The basic technique is to study the behavior of the partially massless field near the boundary of AdS space. This was done recently in [i, with a different motivation. In this section, we assume $D=4$ and $d=3$.

To solve ( $2 . \overline{2}$ ), we impose a gauge condition $\phi_{\mu}^{\mu}=0$, and use the constraint $D^{\mu} \phi_{\mu \nu}=0$ to reduce the equation in $D=4$ to

$$
\left(\square-\frac{4 \Lambda}{3}\right) \phi_{\mu \nu}-2 C_{\alpha \mu \nu \rho} \phi^{\alpha \rho}=0 \text {. }
$$

Following [i], 还, we recall the traceless-transverse decompositions for vectors and tensors

$$
\begin{aligned}
\phi_{\mu}^{T}= & \phi_{\mu}-D_{\mu} \frac{1}{\square} D \cdot \phi, \quad D \cdot \phi^{T}=0, \\
\phi_{\mu \nu}^{T T}= & \phi_{\mu \nu}-D_{(\mu} \frac{2}{\square+\Lambda}\left(D \cdot \phi_{\nu)}\right)^{T}-\frac{1}{4} g_{\mu \nu} \phi_{\rho}^{\rho}- \\
& -D_{[\mu} D_{\nu]} \frac{4}{\square(3 \square+4 \Lambda)} 0\left[D \cdot D \cdot \phi-\frac{1}{4} \square \phi_{\rho}^{\rho}\right], \\
D \cdot \phi_{\mu \nu}^{T T}= & 0=\phi_{\rho}^{T T \rho},
\end{aligned}
$$

where $[\cdots]$ on tensor indices denotes the symmetric traceless part $M_{[\mu \nu]} \equiv M_{(\mu \nu)}-$ $\frac{1}{4} g_{\mu \nu} M_{\rho}^{\rho}$, and $\square \equiv D^{\mu} D_{\mu}$ as usual. We define the spatially traceless-transverse part $\phi_{i j} \equiv \phi_{i j}^{T T}$. The solution [i1] that is more singular near $u=0$ is

$$
\phi_{i j}(u, x) \sim u^{-1} \phi_{0 i j}(x)
$$

so that

$$
\phi_{i}^{j}(u, x)=g^{j k} \phi_{i k} \sim u \phi_{0 i}{ }^{j}(x) .
$$

In the AdS/CFT correspondence, a bulk field $\phi$ that behaves near the boundary as

$$
\phi_{i}{ }^{j}(u, x)=u^{d-h} \phi_{0 i}{ }^{j}(x)
$$




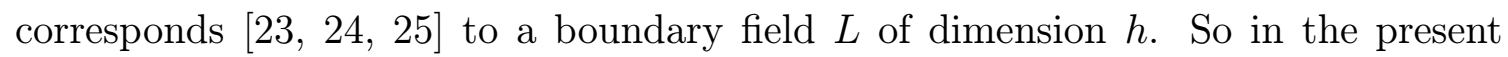
case, $L$ has conformal dimension two, as expected on the basis of the conformally invariant partial conservation law (2., $\bar{k})$. Note that a second independent solution would replace (3.3. quantization and result in the scaling dimension of 1 instead of 2.)

By proceeding with more care, we can actually derive the law (12.6i). We write ${ }^{4} D$ and ${ }^{3} D$ for four- and three-dimensional covariant derivatives and similarly for the affine connections. Using the metric (2.2. $\left.\overline{4}_{1}\right)$, we evaluate the gauge transformation law $\left(\underline{2} . \overline{3}_{-}^{\prime}\right)$ on $\phi_{i j}(u, x)$ near the boundary as

$$
\begin{aligned}
\left({ }^{4} D_{i}{ }^{4} D_{j}+\frac{\Lambda}{3} g_{i j}\right) \xi= & \left(\partial_{i} \partial_{j}-{ }^{4} \Gamma^{k}{ }_{i j} \partial_{k}-{ }^{4} \Gamma^{u}{ }_{i j} \partial_{u}+\frac{\Lambda}{3} g_{i j}\right) \xi \\
\sim & -\tilde{g}_{i j}\left(u^{-2} \xi+u^{-1} \partial_{u} \xi\right)+ \\
& +\left({ }^{3} D_{i}{ }^{3} D_{j}+(d-2)^{-1}\left(\tilde{R}_{i j}-\frac{1}{2(d-1)} \tilde{R} \tilde{g}_{i j}\right)\right) \xi
\end{aligned}
$$

since to order $O\left(u^{2}\right)$ we find ${ }^{4} \Gamma^{k}{ }_{i j} \sim{ }^{3} \Gamma^{k}{ }_{i j}$ and ${ }^{4} \Gamma^{u}{ }_{i j} \sim u^{-1} \tilde{g}_{i j}$. Near the boundary,

$$
\phi_{0 i j}(x)=u \phi_{i j}(u, x)
$$

is independent of $u$ so we take the scalar gauge parameter to behave as $\xi(u, x) \sim$

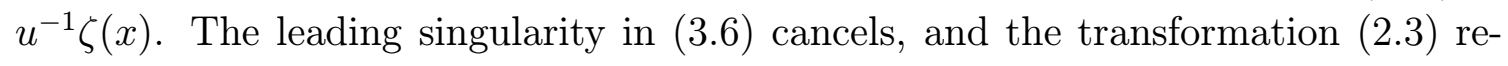
stricted to the boundary is

$$
\phi_{0 i j}=u \phi_{i j}(u, x) \longrightarrow \phi_{0 i j}+\left(D_{i} D_{j}+(d-2)^{-1}\left(\tilde{R}_{i j}-\frac{1}{2(d-1)} \tilde{R} \tilde{g}_{i j}\right)\right) \zeta
$$

In the AdS/CFT equivalence, each field propagating on the AdS space is paired with an operator in the conformal field theory. We consider adding to the lagrangian a Weyl invariant term $\int d^{3} x \sqrt{|\tilde{g}|} L^{i j} \phi_{0 i j}$ that couples the partially massless field to a conformal operator $L^{i j}$. Requiring the invariance of this term under the transformation (3... parts that

$$
\int d^{3} x \sqrt{|\tilde{g}|}\left(D_{i} D_{j} L^{i j}+(d-2)^{-1} \tilde{R}_{i j} L^{i j}\right) \zeta=0
$$

for all $\zeta$. This gives $\left(\underline{2} . \overline{6}_{-}^{\prime}\right)$.

\section{Conformal algebra and unitary conformal field theory}

Now we turn to study the partially conserved conformal tensor $L$ from the point of view of conformal field theory and representations of the conformal algebra. For this purpose, we take the boundary to be flat and use radial quantization. Weyl invariance insures invariance under $H=\mathrm{SO}(d+1,1)$, the global euclidean conformal 
symmetry group of $d$-dimensional flat space. The generators of $H$ are rotations $M_{i j}$, translations $P_{i}$, special conformal transformations $K_{i}$, and dilatations $D$, for $1 \leq i \leq d$, with non-zero commutation relations

$$
\begin{aligned}
{\left[M_{i j}, M_{r s}\right] } & =\delta_{i r} M_{j s}-\delta_{i s} M_{j r}-\delta_{j r} M_{i s}+\delta_{j s} M_{i r} \\
{\left[M_{i j}, P_{r}\right] } & =\delta_{i r} P_{j}-\delta_{j r} P_{i}, \quad\left[M_{i j}, K_{r}\right]=\delta_{i r} K_{j}-\delta_{j r} K_{i}, \\
{\left[D, K_{i}\right] } & =-K_{i}, \quad\left[D, P_{i}\right]=P_{i}, \quad\left[K_{i}, P_{j}\right]=\delta_{i j} D+M_{i j} .
\end{aligned}
$$

Using this conformal algebra, we will rederive the conformal dimension of the field $L$ by requiring that a certain descendant of the state corresponding to $L$ is a null vector We will also show that the first descendant state has negative norm.

As reviewed in [26] given by $D$, and a symmetry algebra $\mathrm{SO}(d+1,1)$ whose generators satisfy hermiticity properties $M_{i j}^{\dagger}=-M_{i j}, P_{i}^{\dagger}=K_{i}, K_{i}^{\dagger}=P_{i}, D^{\dagger}=D$. The generators $M_{i j}=-M_{j i}$ form an $\mathrm{SO}(d)$ subalgebra. Conformal operators are in one to one correspondence with the states of the conformal field theory, $\lim _{x \rightarrow 0} \mathcal{O}(x)|0>=| \mathcal{O}>$. These are eigenstates of the dilatation operator $D$, with eigenvalue given by the scaling dimension. The generators $P_{i}\left(K_{i}\right)$ raise (lower) the scaling dimension, and irreducible representations of the conformal algebra each contain a primary operator $L(x)$ which satisfies $\left[K_{i}, L(0)\right]=0$. Other states in the representation correspond to descendants $\left[P_{i_{1}},\left[P_{i_{2}}, \ldots\left[P_{i_{k}}, L(0)\right] \ldots\right]\right]$.

For clarity, along with the partially conserved tensor $L^{i j}$, we will also consider an ordinary conserved tensor $T^{i j}$ (the usual example is the stress tensor). $T^{i j}$ obeys an ordinary conservation law $\partial_{i} T^{i j}=0$. This corresponds to vanishing of the operator $\left[P_{i}, T^{i j}\right]$ or equivalently of the state $P_{i}\left|T^{i j}\right\rangle$. Similarly, a partially conserved tensor $L^{i j}$ obeys eq. $\left(2, \overline{6}^{\prime}\right)$ which in flat space reduces to $\partial_{i} \partial_{j} L^{i j}=0$. This corresponds to vanishing of the operator $\left[P_{i},\left[P_{j}, L^{i j}\right]\right]$ or the state $P_{i} P_{j}\left|L^{i j}\right\rangle . L$ and $T$ are both traceless and symmetric. The states $\left|T^{i j}\right\rangle$ and $\left|L^{i j}\right\rangle$ are primaries, with

$$
K_{s}\left|T^{i j}\right\rangle=K_{s}\left|L^{i j}\right\rangle=0
$$

The transformation law of $\left|T^{i j}\right\rangle$ under rotations is

$$
M_{r s}\left|T_{i j}\right\rangle=\delta_{r i}\left|T_{s j}\right\rangle+\delta_{r j}\left|T_{i s}\right\rangle-\delta_{s i}\left|T_{r j}\right\rangle-\delta_{s j}\left|T_{i r}\right\rangle,
$$

and similarly for $\left|L^{i j}\right\rangle$. To begin with, we denote as $h$ the conformal dimension of $T$ and $L$ and do not assume that they are conserved or partially conserved.

For the states $P_{i}\left|T^{i j}\right\rangle$ and $P_{i} P_{j}\left|L^{i j}\right\rangle$ to vanish, their norms must be zero. These norms can be evaluated as follows, using ( $\left(\bar{A}_{-} \overline{2}_{1}^{\prime}\right)$. For $T$ we have

$$
\begin{aligned}
\left.\|\left|P_{i}\right| T^{i j}\right\rangle \|^{2} & =\left\langle P_{s} T^{r s} \mid P_{i} T^{i j}\right\rangle=\left\langle T^{r s} \mid K_{s} P_{i} T^{i j}\right\rangle \\
& =\left\langle T^{r s} \mid\left[K_{s}, P_{i}\right] T^{i j}\right\rangle=\left\langle T^{r s} \mid\left(\delta_{s i} D+M_{s i}\right) T^{i j}\right\rangle \\
& =(h-d)\left\langle T^{r s} \mid T_{s}^{j}\right\rangle,
\end{aligned}
$$




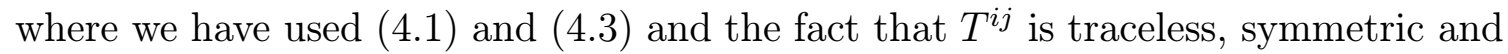
primary with scaling dimension $h$. From $\left(\bar{A}_{-} \bar{A}_{1}^{\prime}\right)$, we have that $P_{i} \mid T^{i j}>$ is a null state precisely if $h=d$. This result was to be expected, since $d$ is the standard value of the scaling dimension of the stress tensor in $d$ spacetime dimensions. We also see that $P_{i}\left|T^{i j}\right\rangle$ has negative norm if $h<d$.

Evaluating similar commutators, we find

$$
\begin{aligned}
\| P_{i} P_{j}\left|L^{i j}\right\rangle \|^{2} & =\left\langle L^{r s} \mid K_{r} K_{s} P_{i} P_{j} L^{i j}\right\rangle \\
& =\left\langle L^{r s} \mid K_{r}\left[K_{s}, P_{i}\right] P_{j} L^{i j}\right\rangle+\left\langle L^{r s} \mid K_{r} P_{i}\left[K_{s}, P_{j}\right] L^{i j}\right\rangle \\
& =2(h-(d-1))\left\langle L^{r s} \mid K_{r} P_{j} L^{i j}\right\rangle \\
& =2(h-(d-1))(h-d)\left\langle L^{r s} \mid L_{r s}\right\rangle .
\end{aligned}
$$

So for $P_{i} P_{j}\left|L^{i j}\right\rangle$ to be a null vector, we require $h=d-1$, as found earlier.

Applying the same technique, we can show that the first descendant of $L$ is actually a state of negative norm. In fact,

$$
\|\left|P_{i} L^{i j}\right\rangle \|^{2}=-\left\langle L^{j s} \mid L_{s}^{j}\right\rangle .
$$

The computation here is precisely the same as the one in ( reflects the fact that the conformal dimension of $L$ is less than that of $T$. In terms of partially massless fields in AdS space, this corresponds directly to the sign of the formulas for $m^{2}$ in (1;. $\left.\overline{1}_{1}^{\prime}\right)$. Thus, as mentioned in the introduction, the physical properties of partially massless fields in de Sitter space may be better.

What property of the partially massless field $\phi$ in AdS space corresponds to the negative norm of the first descendant of $L$ on the boundary? The descendants of $L$ all have real conformal dimensions, greater than that of $L$, but one of them has negative norm. This seems to mean that $\phi$ can be quantized with energies bounded below - and with the Fock ground state having the smallest energy — but not in a Hilbert space with all states having positive norm. This is a delicate result that will depend upon analysis of global boundary conditions in AdS space.

Though we have restricted ourselves to fields of spin 2 up to this point, the computations in this section are readily generalized to an $s^{\text {th }}$ rank symmetric traceless tensor $L^{i_{1} i_{2} \cdots i_{s}}$. Looking for null vectors that would correspond to a partial conservation law

$$
\partial_{i_{1}} \cdots \partial_{i_{m}} L^{i_{1} i_{2} \cdots i_{s}}=0
$$

we get

$$
\begin{aligned}
\|\left|P_{i_{1}} P_{i_{2}} \cdots P_{i_{m}} L^{i_{1} i_{2} \cdots i_{s}}\right\rangle \|^{2}= & \left\langle L^{j_{1} j_{2} \cdots j_{s}}\left|K_{j_{1}} K_{j_{2}} \cdots K_{j_{m}} P_{i_{1}} P_{i_{2}} \cdots P_{i_{m}}\right| L^{i_{1} i_{2} \ldots i_{s}}\right\rangle \\
= & m !(h-(d+s-2))(h-(d+s-3)) \cdots \\
& \left.\cdots(h-(d+s-m-1))\|\| L^{i_{1} i_{2} \cdots i_{s}}\right\rangle \|^{2}
\end{aligned}
$$


If we want (A. every partial conservation law obeyed by $L$ is a consequence of ('A $\left.\bar{A}_{-} \bar{Z}_{-}^{\prime}\right)$, we must take $h=d+s-r-1$. Such an $L$ should correspond in AdS space to a partially massless field with a range of helicities missing, depending on $r$. The first descendant always has negative norm, if $r>1$.

\section{A cosmological solution}

Since we do not have a full knowledge of the elementary particles, it might be that in addition to the usual massless particles such as photons whose interactions are trivial in the infrared, there are additional massless modes with infrared-non-trivial interactions, governed by a non-trivial conformal field theory. If we neglect the problem with the negative norm descendant (as we will in the present section), then we can imagine that this theory might admit a partially conserved $L$ tensor such as we have studied above. If so, to characterize the physical state of the expanding universe, in addition to the usually almost conserved quantities such as baryon number, energy, and entropy, one must also specialize the value of the $L$ tensor.

To get an idea of cosmology with the $L$ tensor, we will solve the partial conservation eq. (12.6. $\left.{ }^{\prime}\right)$ in the background of a $d$-dimensional Robertson-Walker metric with flat spatial sections:

$$
\tilde{g}_{i j} d x^{i} d x^{j}=d t^{2}-f^{2}(t) \delta_{m n} d x^{m} d x^{n} .
$$

We assume that the $L$ tensor is invariant under the spatial rotation and translation symmetries of the background solution. This implies that $L^{00}$ is a function of time only, that $L^{0 m}=0$, and (as $L$ is traceless) that $L^{m n}=\delta^{m n}(d-1)^{-1} f^{-2} L^{00}$. Solving the partial conservation equation, we will express $L^{00}$ in terms of the conformal time $\eta=\int^{t} d t^{\prime} f^{-1}\left(t^{\prime}\right)$. For the metric (15. $\left.\overline{1}_{1}^{\prime}\right)$, the non-vanishing components of the Ricci tensor are $\tilde{R}_{00}=-(d-1) f^{-1} \partial_{0}^{2} f$ and $\tilde{R}_{m n}=\delta_{m n}\left((d-2)\left(\partial_{0} f\right)^{2}+f \partial_{0}^{2} f\right)$. Then $\tilde{R}_{i j} L^{i j}=-(d-2)\left(\partial_{0} H\right) L^{00}$ where $H$ is the Hubble function $H(t) \equiv f^{-1} \partial_{0} f$, and the

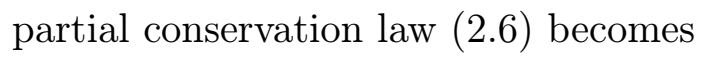

$$
\partial_{0}^{2} L^{00}+(2 d-1) H \partial_{0} L^{00}+(d-1)\left(\partial_{0} H\right) L^{00}+d(d-1) H^{2} L^{00}=0 .
$$

We can factor this as

$$
\left(\partial_{0}+d H\right)\left(\partial_{0}+(d-1) H\right) L^{00}=0,
$$

and solve it by defining $G(t) \equiv \partial_{0} L^{00}+(d-1) H L^{00}$, where $\partial_{0} G=-d H G$. Then either $G=0$, or $G=e^{-d \int^{t} d t^{\prime} H\left(t^{\prime}\right)}=f^{-d}$. For $G=0$, we have $L^{00}=e^{-(d-1) \int^{t} d t^{\prime} H\left(t^{\prime}\right)}=$ $f^{-(d-1)}$. For the second solution, when $G=f^{-d}$ then $L^{00}$ can be expressed as $f^{-(d-1)} \eta$ where $d \eta \equiv d t f^{-1}(t)$

$$
L^{00}(t)=(f(t))^{-(d-1)} \int^{t} d t^{\prime} f^{-1}\left(t^{\prime}\right) .
$$


The unique general solution of $\left(\overline{2}_{2} . \overline{6}^{\prime}\right)$ in terms of two arbitrary constants $a_{1}, a_{2}$ for the partially conserved rotationally symmetric boundary operator is

$$
L^{00}=f^{-(d-1)}\left(a_{1}+a_{2} \eta\right)
$$

In the spirit of the partial conservation law $\left(12 . \overline{6} \overline{6}_{1}^{\prime}\right)$, we can identify the partially conserved charge

$$
\begin{aligned}
Q & \equiv \int d^{d-1} x \sqrt{\tilde{g}} D_{i} L^{i 0}=\int d^{d-1} x f^{(d-1)}\left(\partial_{0} L^{00}+d H L^{00}\right) \\
& =\left(\int d^{d-1} x\right)\left(a_{1} H+a_{2}\left(H \eta+f^{-1}\right)\right),
\end{aligned}
$$

where $\partial_{0} Q \neq 0$ but

$$
\partial_{0} Q=-(d-2)^{-1} \int d^{d-1} x \sqrt{\tilde{g}} \tilde{R}_{i j} L^{i j}=\left(\int d^{d-1} x\right)\left(a_{1}+a_{2} \eta\right) \partial_{0} H,
$$

which is an identity on shell since $H \partial_{0} \eta+\partial_{0} f^{-1}=0$.

In standard cosmology, the energy-momentum tensor $T_{j}^{i}=\operatorname{diag}(\rho,-p,-p,-p)$ provides the evolution of the cosmic scale factor $f(t)$ from the Einstein field equations as

$$
f(t) \sim t^{2 /(3(1+\omega))},
$$

where $\rho \sim f^{-3(1+\omega)}$ and $\rho=\omega p$, for $\omega$ independent of time. The standard examples include photons $f \sim t^{1 / 2}$ for $\omega=1 / 3$; matter $f \sim t^{2 / 3}$ with $\omega=0$; de Sitter inflation $f \sim e^{t}$ for $\omega=-1$; and a curvature dominated model $f \sim t$ with $\omega=-1 / 3$. The explicit form of the cosmological scale factor enables one to study the rate of speeding up of the expansion: the acceleration parameter $q(t) \equiv\left(\partial_{0} f\right)^{-2} \partial_{0}^{2} f f$ is negative for the first two examples, positive for the de Sitter universe, and zero for the last example.

Thus, we have shown that in the context of cosmology, the partial conservation law determines the time-dependence of the $L$-field, which evolves according to a quasi-conservation law somewhat analogous to that of more familiar almost conserved quantities in cosmology.

\section{Acknowledgments}

All authors thank the Caltech-USC Center for Theoretical Physics where this work was initiated. CRN was partially supported by the U.S. Department of Energy, Grant No. DE-FG03-84ER40168. Research of EW was supported in part by NSF Grant PHY-0070928 and the Caltech Discovery Fund. LD also thanks the Institute for Advanced Study at Princeton for its hospitality, and was partially supported by the U.S. Department of Energy, Grant No. DE-FG02-97ER-41036/Task A. 


\section{References}

[1] S. Deser and A. Waldron, Null propagation of partially massless higher spins in $(A) d S$ and cosmological constant speculations, iphys. Lett. B [hep-th/0105181i.

[2] S. Deser and A. Waldron, Partial masslessness of higher spins in (A)dS, iNucucl. Phys.:

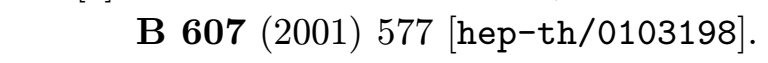

[3] S. Deser and R. Nepomechie, Gauge invariance versus masslessness in de Sitter spaces,

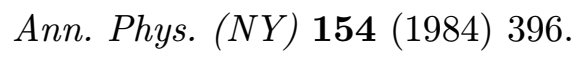

[4] S. Deser and R. Nepomechie, Anomalous propagation of gauge fields in conformally flat spaces, יㅡㄴ

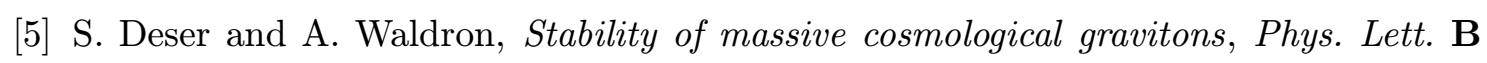
(-

[6] S. Deser and A. Waldron, Gauge invariances and phases of massive higher spins in

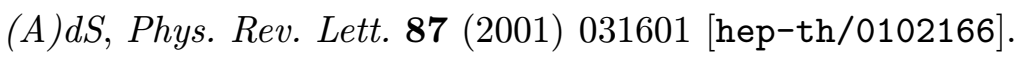

[7] A. Higuchi, Massive symmetric tensor field in spacetimes with a positive cosmological constant, iNucl. Phys. B $\overline{3} \mathbf{2} \overline{5}(\overline{1} \overline{9} \overline{9}) \overline{4} \overline{4}$.

[8] A. Higuchi, Forbidden mass range for spin-2 field theory in de Sitter spacetime, Nㅡㄴ :

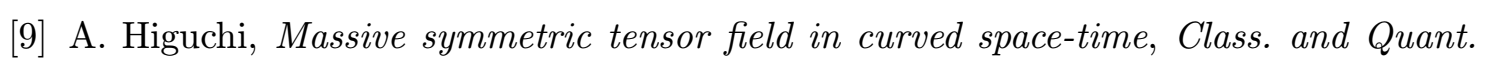
Grav.

[10] A. Higuchi, Symmetric tensor spherical harmonics on the $N$-sphere and their applica-

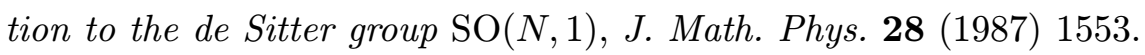

[11] I.L. Buchbinder, D.M. Gitman and V.D. Pershin, Causality of massive spin 2 field in

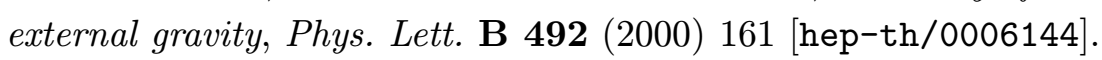

[12] I.L. Buchbinder, D.M. Gitman, V.A. Krykhtin and V.D. Pershin, Equations of motion for massive spin 2 field coupled to gravity, ${ }_{2}$ ucl. Phys. [hep-th/9910188i'.

[13] M. Eastwood, Notes on conformal differential geometry, (available electronically: ftp://ftp.maths.adelaide.edu.au/pure/meastwood/srni95.ps), Suppl. Rendi. Circ. Mat. Palermo 43 (1996) 57.

[14] R.J. Baston and M. Eastwood, Invariant operators, in the collection Twistors in Mathematics and Physics, L.M.S. Lecture Notes 156, C.U.P. 1990, pp. 129.

[15] M. Eastwood and J.W. Rice, Conformally invariant differential operators on

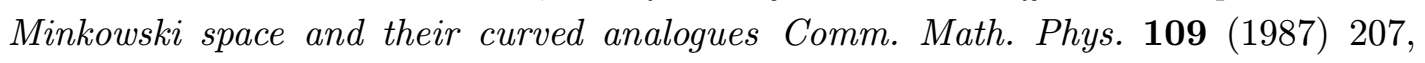
erratum ibid. 144 (1992) 213. 
[16] M. Eastwood and J. Slovak, Semi-holonomic Verma modules, Jour. Alg. 197 (1997) 424.

[17] E. Witten, Quantum gravity in de Sitter space, hep-th/010610

[18] A. Strominger, The dS/CFT correspondence, hep-th/0106113.

[19] C. Fefferman and C.R. Graham, Conformal invariants, in Elie Cartan et les mathmiatiques d'aujourdhui, Asterisque, 1985, p. 95.

[20] M. Henningson and K. Skenderis, The holographic Weyl anomaly, 'J. High Energy' Phys. 07 (1998) 023i lihep-th/9806087!.

[21] M. Henningson and K. Skenderis, Holography and the Weyl anomaly, Fortsch. Phys. 48 (2000) 125 [hep-th/9812032i].

[22] C.R. Graham and E. Witten, Conformal anomaly of submanifold observables in

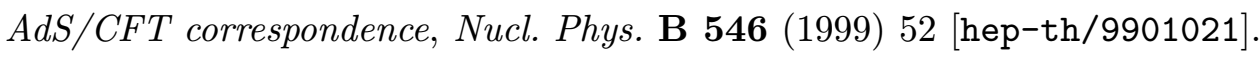

[23] S.S. Gubser, I.R. Klebanov and A.M. Polyakov, Gauge theory correlators from non-

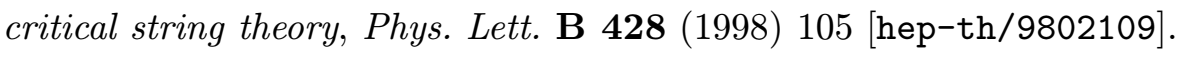

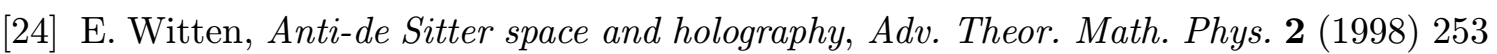
[hep-th/9802150i'.

[25] O. Aharony, S.S. Gubser, J. Maldacena, H. Ooguri and Y. Oz, Large-N field theories, string theory and gravity, 'Phys. Rep $\mathbf{3} \overline{2}$ - $(2000)$ 183' [hep-th/9905111].

[26] S. Minwalla, Restrictions imposed by superconformal invariance on quantum field theories, "Ádv. Theor. Math Phys. 2 (1998) 781' (hep-th/9712074t. 